\title{
HIGHLIGHT
}

\section{Cushing's syndrome: from patients to proteins}

\author{
Mansur E Shomali and Mehboob A Hussain ${ }^{1}$ \\ Endocrine Unit and ${ }^{1}$ Laboratory of Molecular Endocrinology, Howard Hughes Medical Institute, Massachusetts General Hospital, \\ Harvard Medical School, Boston, Massachusetts 02114, USA; Fax: +1-617-7266954
}

In 1932, Harvey Cushing reviewed the medical history of Minnie $\mathrm{G}$ and 11 other patients with a rapid weight gain confined to the face, neck, and trunk, but sparing the extremities (1). This adiposity was associated with kyphosis, amenorrhea or impotence, hirsutism, purplish lineae atrophicae, hypertension, and extreme weakness. Cushing concluded that the peculiar polyglandular syndrome may accompany basophil adenoma in the absence of any apparent alteration in the adrenal cortex other than a possible secondary hyperplasia.'

Cushing's reasoning was correct. The majority of patients with the syndrome that now bears his name suffer from adrenal hypersecretion of cortisol due to excess of corticotropin (ACTH). The basophilic cells in Cushing's series were most likely expansions of the population of pituitary corticotropes. But what is the pathogenesis of ACTH hypersecretion? Why do some 'silent' pituitary tumors show histochemical evidence for ACTH production but do not hypersecrete it? And why are not all ACTH-secreting tumors associated with atrophy of the neighboring normal corticotropes which should be suppressed? Current research has begun to elucidate the answers to some of these questions.

The anterior pituitary corticotropes produce ACTH by processing products of the pro-opiomelanocortin (POMC) gene (see Fig. 1). Up to $10 \%$ of normal subjects may harbor small, POMC-containing pituitary adenoma (2). These adenoma are endocrinologically silent, presumably due to production of inactive POMC peptides or peptides that are not secreted. In patients with Cushing's Disease, the frequency of identifiable pituitary tumors is much greater. However, the pathological findings are sometimes classified as corticotrope hyperplasia rather than adenoma. This has led to two major outlooks on the pathogenesis of Cushing's disease, i.e. the hypothalamic theory and the pituitary theory (3). Evidence favoring the hypothalamic theory is that many histopathological specimens are classified as hyperplasia rather than adenoma. Of course, this may be due to difficulty in interpreting pathology of small tumor fragments. However, excess corticotropin-releasing hormone (CRH) has been shown to produce Cushing's disease in patients with ectopic CRH production and in transgenic mice that overexpress the $\mathrm{CRH}$ gene (4). Evidence favoring the pituitary theory, which proposes that mutated corticotropes give rise to neoplastic clones that produce excess ACTH, is that permanent cure results from successful resection of pituitary tumors in the majority of patients. Successfully treated patients regain normal circadian rhythms for ACTH and other pituitary hormones. The factors that may lead to the development of corticotrope neoplasms, whether entirely of pituitary or perhaps of hypothalamic origin, are yet to be identified.

Although most corticotrope neoplasms are believed to arise from the anterior pituitary, there have been patients with ACTH-secreting tumors whose origins seem to have been from the intermediate lobe (2). In humans, the intermediate lobe is a rudimentary structure; however, in other species it is well developed and functions to regulate skin pigmentation. Intermediate lobe melanotropes produce $\alpha$-melanocyte-stimulating hormone ( $\alpha$-MSH), an alternative splice product of POMC, which is generated by tissue-specific enzyme cleavage. The enzyme prohormone convertase 2 (PC2), a member of the proprotein convertase family of enzymes $(5,6)$, is expressed in the intermediate lobe where it cleaves ACTH to $\alpha$-MSH and corticotropin-like intermediate lobe peptide (CLIP). This enzyme is not present in the anterior lobe where another convertase, $\mathrm{PC} 1$, is involved in the processing of POMC. Therefore, in order to produce Cushing's disease, neoplastic melanotropes of the intermediate lobe need to be able to produce sufficient POMC which is aberrantly processed to intact ACTH and then secreted.

Regulation of the POMC gene differs in the two pituitary lobes. Anterior lobe expression of the POMC gene is upregulated by $\mathrm{CRH}$ and inhibited by glucocorticoids. In contrast, melanotrope production of POMC is known to be under negative regulation by dopamine and is not sensitive to glucocorticoid feedback. Mice deficient in the dopamine D2 receptor have intermediate lobe hypertrophy and increased POMC expression; surprisingly they also develop a Cushing's-like syndrome from excess release of ACTH (7). Therefore, not only does loss of dopaminergic inhibition result in POMC overexpression, but processing must also be aberrant in these animals. In fact, the authors report that PC1 is overexpressed in the intermediate lobes of the D2 receptor-deficient animals, which may account for the overproduction of ACTH.

Recently, Westphal et al. reported that mice with a null mutation in the neuroendocrine protein 7B2 develop a Cushing's-like syndrome from intermediate lobe ACTH hypersecretion (8). Via differences in substrate preference and tissue distributions, PC1 and PC2 
POMC precursor

$$
1
$$

anterior lobe processing

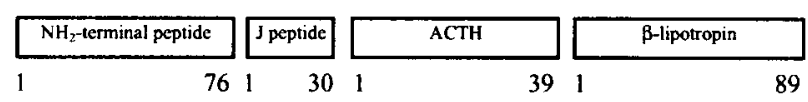

intermediate lobe processing
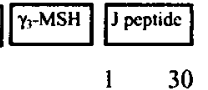

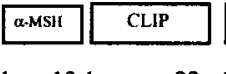

131

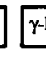

$\gamma$-lipotropin

221
561
Figure 1 Alternate processing of POMC in the anterior and intermediate lobes of the pituitary gland. The pro-opiomelanocortin gene encodes a precursor protein of 241 amino acids, which is alternately spliced into mature peptides. Processing of the precursor protein in the anterior lobe of the pituitary gland, where the enzyme PC- 1 is expressed, leads to the production of ACTH. The intermediate lobe, which expresses PC-2, produces $\alpha$-MSH. are involved in the selective processing of many precursor hormones in addition to $\operatorname{POMC}(5,6) . \mathrm{PC} 2$ differs from the other convertases in that its activity requires interaction with another neuroendocrine protein, 7B2. This protein is thought to act as an intracellular chaperone for PC2, to facilitate its maturation, and to be required for its enzymatic activity (9). In the late endoplasmic reticulum 7B2 binds to folded proPC2, and this complex is transported to the Golgi at a higher rate than proPC2 alone (10). 7B2-bound proPC2 can then undergo cleavage and activation when it reaches secretory granules with the prerequisite low $\mathrm{pH}$ and high calcium concentration. Although proPC2 may be cleaved in the absence of 7B2, this form is, for unknown reasons, not active (6).

In order to better study the role of 7B2, Westphal \& Leder (11), using a novel transposon-based technology, generated 7B2 null mice. They predicted that these mice would be deficient in PC2 activity, and they were correct. PC2 participates in the processing of proinsulin and proglucagon in the islets of Langerhans, and PC2deficient mice have impaired processing of these hormones, resulting in chronic fasting hypoglycemia and a reduced rise in blood glucose levels during a glucose tolerance test (12). 7B2 null mice were also deficient in processing these hormones and were also hypoglycemic. In contrast to the PC2 mice, these animals exhibit many of the features of Cushing's syndrome, i.e. severe bruising, thinning of the skin, abnormal fat deposition on the back and around the neck, and a fourfold elevation of circulating corticosterone. The pituitaries of these animals have a 10- to 20-fold elevation of intact ACTH and minimal $\alpha$-MSH. Anterior lobe ACTH immunostaining is absent, suggesting that the excess ACTH is derived from the intermediate lobe. Anterior lobe, but not intermediate lobe, POMC expression is downregulated by glucocorticoids, thus accounting for the absence of anterior lobe ACTH immunostaining. Although PC2-deficient mice pituitaries also have elevated ACTH (presumably due to impaired processing of intermediate lobe POMC), they do not develop a Cushing's-like syndrome and corticosterone levels are normal.

Westphal et al. speculate that 7B2 may have additional roles in neurosecretory function not related to PC2-mediated effects. In fact, 7B2 mRNA is widely distributed in neuronal and endocrine tissue, much more than the more limited distribution of PC2 mRNA (13). Preliminary results show that isolated pituitaries from these animals have a greatly enhanced secretion of intact ACTH, suggesting that these pituitaries have intrinsic hypersecretion, even when removed from their usual regulatory influences. As a distinct function, 7B2 may be important for modulating secretion from neuroendocrine cells. This may account for the increase release of ACTH into the circulation of $7 \mathrm{~B} 2$ null compared to PC2 null mice. A disease of hormonal excess, therefore, may be due to an isolated problem of aberrant hormonal secretion, and studying the control of ACTH release in these mice may provide further insight into other diseases involving secretory hormones. Such studies may lead to an explanation of how some 'non-functional' pituitary tumors may produce their hormones in excess, but not lead to increased levels of the hormone in the circulation.

The proximate cause of the metabolic abnormalities in Cushing's syndrome is adrenal hypersecretion of cortisol, which may sometimes be due to a primary adrenal problem. In this case, the hypercortisolism is ACTH-independent. These patients usually present with either a single benign (or rarely malignant) adrenal tumor. Occasionally, bilateral macronodular or micronodular adrenocorticol hyperplasia occurs. Although the pathogenesis of these disorders is incompletely understood, exciting new insights are being gleaned from some specific entities. Primary pigmented nodular adrenocortical disease (PPNAD) is a rare cause of hypercortisolism that is often associated with the familial multiple neoplasia syndrome known as the Carney complex (14). Linkage analysis of affected families has identified a locus on the short arm of chromosone 2 . Identification of the aberrant gene in this locus and studying its role in tumorogenesis should lead to a greater understanding of the biology of adrenal tumors.

An activating somatic mutation of the $\mathrm{G}$ protein, Gs $\alpha$, which occurs in McCune-Albright syndrome, may result in functional adrenal tumors (15). With this knowledge, one may deduce that abnormalities in cellsurface receptors could also cause hypercortisolism. In fact, multiple $\mathrm{G}$ protein-coupled receptors have been 
identified on adrenocortical cells in vitro, and investigators have found that ACTH-independent Cushing's syndrome may be caused by the modulation of cortisol production by abnormally expressed receptors. These illicit or promiscuous receptors include those for gastric inhibitory peptide, vasopressin, interleukin-1, catecholamines, luteinizing hormone, serotonin, and thyrotropin $(16,17)$. The hypercortisolism in these cases may be treated by a specific receptor antagonist drug, e.g. a $\beta$-blocker in the case of abnormal expression of the $\beta$-adrenergic receptor.

Patients with Cushing's syndrome have very serious metabolic abnormalities due to cortisol hypersecretion. Treating these patients necessitates localization of the source of the hormonal abnormality to the adrenal gland or to the pituitary. Molecular medicine has led to tremendous improvements in the care of these patients and has given us fascinating insights into hormones, receptors and their regulation.

\section{Acknowledgements}

MES is supported by a National Research Service Award from the National Institutes of Health DK10001-01. MAH holds a Juvenile Diabetes Foundation International Career Development Award.

\section{References}

1 Cushing $\mathrm{H}$. The basophil adenomas of the pituitary body and their clinical manifestations (pituitary basophilism). Bulletin of the Johns Hopkins Hospital 1932 L 137-195.

2 Krieger DT. Physiopathology of Cushing's Disease. Endocrine Reviews $1983422-43$.

3 Biller BMK. Pathogenesis of pituitary Cushing's Syndrome: pituitary versus hypothalamic. Endocrinology and Metabolism Clinics of North America 199423 547-554.

4 Stenzel-Poore MP, Cameron VA, Vaughan J, Sawchenko PE \& Vale W. Development of Cushing's syndrome in corticotropinreleasing factor transgenic mice. Journal of Clinical Endocrinology and Metabolism 1992130 3378-3386.

5 Seidah NG \& Chrétien M. Proprotein and prohormone convertases: a family of subtilases generating diverse bioactive polypeptides. Brain Research 1999848 45-62.
6 Canaff L, Bennett HPJ \& Hendy GN. Peptide hormone precursor processing: getting sorted? Molecular and Cellular Endocrinology $19991561-6$.

7 Saiardi A \& Borrelli E. Absence of dopaminergic control on melanotrophs leads to Cushing's like syndrome in mice. Molecular Endocrinology 199812 1133-1139.

8 Westphal CH, Muller L, Zhou A, Zhu X, Bonner-Weir S, Schambelan M, Steiner DF, Lindberg I \& Leder P. The neuropeptide $7 \mathrm{~B} 2$ is required for peptide hormone processing in vivo and provides a novel mechanism for pituitary Cushing's disease. Cell $199996689-700$.

9 Zhu X \& Lindberg I. 7B2 facilitates the maturation of proPC2 in neuroendocrine cells and is required for the expression of enzymatic activity. Journal of Cell Biology $19951291641-$ 1650.

10 Muller L, Zhu X \& Lindberg I. Mechansim of the facilitation of PC2 maturation by 7B2: involvement in proPC2 transport and activation but not folding. Journal of Cell Biology 1997139 625638.

11 Westphal CW \& Leder P. Transposon-generated 'knock-out' and 'knock-in' gene-targeting constructs for use in mice. Current Biology 19977 530-533.

12 Furuta M, Yano H, Zhou A, Rouille Y, Holst JJ, Carroll R, Ravazzola M, Orci L, Furuta H \& Steiner DF. Defective prohormone processing and altered pancreatic islet morphology in mice lacking active SPC2. PNAS 199794 6646-6651.

13 Seidel B, Dong W, Savaria D, Zheng M, Pintar JE \& Day R. Neuroendocrine protein $7 \mathrm{~B} 2$ is essential for proteolytic conversion and activation of proprotein convertase 2 in vivo. DNA and Cell Biology 199817 1017-1029.

14 Stratakis CA, Carney JA, Lin JP, Papanicolaou DA, Karl M, Kastner DL, Pras E \& Chrousos GP. Carney Complex, a familial multiple neoplasia and lentiginosis syndrome: analysis of 11 kindreds and linkage to the short arm of chromosome 2. Journal of Clinical Investigation 199697 699-705.

15 Ringel, MD, Schwindinger WF \& Levine MA. Clinical implications of genetic defects in $\mathrm{G}$ proteins: the molecular basis of McCuneAlbright Syndrome and Albright Hereditary Osteodystrophy. Medicine 199675 171-184.

16 Willenberg HS, Stratakis CA, Marx C, Ehrhart-Bornstein M, Chrousos GP \& Bornstein SR. Aberrant interleukin-1 receptors in a cortisol-secreting adrenal adenoma causing Cushing's syndrome. New England Journal of Medicine 1998339 27-31.

17 Lacroix A, Mircescu H \& Hamet P. Clinical evaluation of the presence of abnormal hormone receptors in adrenal Cushing's syndrome. Endocrinologist 1999 9 9-15.

Received 20 April 2000

Accepted 11 May 2000 